\title{
On the Creation of Acceptable Conjoint Analysis Experimental Designs
}

\author{
Joel H. Steckel \\ Stern School of Business, New York University, New York, NY 10003 \\ Wayne S. DeSarbo \\ School of Business Administration, University of Michigan, Ann Arbor, MI 48109 \\ Vijay Mahajan \\ Graduate School of Business Administration, University of Texas, Austin, TX 78712
}

\begin{abstract}
Conjoint analysis studies typically utilize orthogonal fractional factorial experimental designs to construct a set of hypothetical stimuli. Occasionally, these designs include environmentally correlated attributes that can lead to stimulus profiles that are not representative of the subject's environment. To date, no one has proposed a remedy well-grounded in statistical theory. This note presents a new methodology utilizing combinatorial optimization procedures for creating modified fractional factorial designs that are as "orthogonal" as possible, which do not contain nonrepresentative stimulus profiles.

Subject Areas: Marketing Research, Product Design and Performance, and Decision Processes.
\end{abstract}

\section{INTRODUCTION}

In the last ten to fifteen years, probably no technique has received more attention towards the modeling of consumer preferences than conjoint analysis [4] [7] [8]. However, some reservations about the technique still exist. In a recent statement, Riedesel [17] argued that the data collected for conjoint studies often do not reflect the constructs being measured. Ironically, some of this difficulty can be attributed to the methodological advances that have made conjoint analysis more accessible. For example, in an effort to reduce the data collection burden on respondents, Green [6] suggested the use of orthogonal fractional factorial experimental designs [1] to construct stimulus sets. Orthogonality guarantees that the resulting parameter estimates obtained from the analysis would have maximum "efficiency" since the attributes (or independent variables in an ANOVA or regression sense) would be devoid of statistical correlation.

On the other hand, Green and Srinivasan [8] argued that substantial environmental correlations between the attributes may exist. Orthogonalizing inherently nonorthogonal attributes is likely to produce stimuli which are not representative of the environment familiar to the subjects. The effect an unfamiliar stimulus has on a subject's response remains unclear. Green and Srinivasan also suggested that subjects may indeed respond unnaturally to nonrepresentative (i.e., unbelievable or unfamiliar) stimuli and that these responses can have an adverse effect on the estimation of conjoint utility functions and any resulting predictions.

Against this background, this paper introduces a new conjoint analysis design generation procedure, grounded in statistical theory, that can create designs of maximum efficiency (i.e., as close as possible to being orthogonal) for a given size (number of stimuli, attributes, and attribute levels) and coding scheme, containing no combinations of attribute levels predetermined to be nonrepresentative. The proposed procedure is fairly simple to use and has been programmed in APL on a microcomputer. The designs created by the proposed methodology can be used 
directly in conjoint analyses. Furthermore, such designs can be used in problems where the researcher wants to eliminate certain alternatives from consideration for any reason whatsoever (e.g., they may be technically infeasible to produce).

It should be noted that in practice, it may be difficult to designate which of a set of profiles are nonrepresentative. Classifying certain stimuli as nonrepresentative is a difficult task that this paper does not address (see [10] for a more complete discussion). In this paper, nonrepresentative stimuli are assumed to be predetermined by whatever method is deemed appropriate for the specific application. A linear main effects part-worth preference model with no interactions is also assumed.

\section{An Example}

As an example, consider a set of preference judgments for automobiles. (This example is a modification of one found in [9].) Assume that the relevant attributes and levels are:

$\begin{array}{ll}\text { Mileage: } & 20 ; 30 ; 40 \mathrm{mpg}, \\ \text { Price: } & \$ 15,000 ; \$ 20,000 ; \$ 25,000, \\ \text { Maximum Speed: } & 85 ; 120 ; 155 \mathrm{mph}, \\ \text { Car Length: } & 10 ; 12 ; 14 \text { feet, } \\ \text { Roominess: } & \text { room for } 4 ; 5 ; 6 \text { passengers. }\end{array}$

The first specific design column of Table 1 presents an eighteen profile orthogonal fraction of the full factorial design which would contain $3^{5}$ or 243 profiles.

Note, however, that there is an environmental correlation among these attributes which could make some of the cars in the table nonrepresentative. For example, suppose a researcher decided that the following attribute level combinations were not representative and were unlikely to be found in the environment:

1. A 14-foot car which could hold 6 passengers and get $40 \mathrm{mpg}$ (the car would be too big to be so fuel efficient);

2. A 14-foot car with maximum speed $155 \mathrm{mph}$ and a cost of only $\$ 15,000$ (the cost of such a car would make such a low price infeasible); and

3. A car with maximum speed $155 \mathrm{mph}$ and mileage $40 \mathrm{mpg}$ (the car's engine would be too large to be so fuel efficient).

While one could arguably question whether such combinations are realistic and/or whether others may not be, we hasten to note that we are just using them for illustrative purposes. As such, these three combinations form constraints around which acceptable designs can be created. Nevertheless, profiles $7,8,13,16$, and 17 in the orthogonal array violate the constraints.

Researchers in consumer decision making have cited a number of ad hoc remedies to the problem of nonrepresentative stimuli in orthogonal designs. These procedures, many of which are discussed in [9], include:

a. Ignore the problem.

b. Search for another orthogonal design.

c. Permute the coding of attribute levels so that the resultant design is representative.

d. Delete the nonrepresentative stimuli.

e. Modify the nonrepresentative profile by changing the level of one or more of the attributes. 
f. Sample stimuli from a multivariate probability distribution that reflects the attributes' means, variances, and correlations.

g. Prepare a composite attribute covering all 'subattributes' with acceptable combinations of attribute levels.

We now introduce a new methodology to handle the nonrepresentative stimulus problem. The methodology centers around the generation of "optimal" experimental designs via a combinatorial optimization procedure accommodating user-specified constraints which correspond to nonrepresentative profiles. The procedure we propose subsumes options $b$ and $c$ above in that if another orthogonal design (without nonrepresentative stimuli) exists, our methodology should find it. Furthermore, the "optimality" of the design guarantees that the parameter estimates are at least as accurate as those provided by options d, e, and f. Finally, unlike in option $\mathrm{g}$, the number of profiles can be kept down to the original number in the orthogonal design.

\section{THE METHODOLOGY}

We are interested in devising a new methodology that will create experimental designs that are as "orthogonal" as possible, but contain no nonrepresentative combinations of specific levels of denoted attributes. The design matrix, $\mathbf{X}$, represents the stimuli that the respondent is judging. Each row represents one stimulus. Each stimulus is defined in terms of its attributes (usually categorized) which are dictated by the columnar entries in that row. The methodology can be employed with any specified coding scheme used in $\mathbf{X}$, including dummy variables, effects coding, contrast coding, and orthogonal coding. In particular, if each attribute can be expressed numerically, as in our automobile example, the actual attribute values can be used.

In conjoint analyses, $\mathbf{X}$ is typically specified via an orthogonal fractional factorial design. In the event that these designs lead to nonrepresentative profiles, one can potentially envision a trade-off between altering the design and accepting some lack of orthogonality versus retaining the orthogonal design and obtaining possibly invalid responses. Our perspective in developing the methodology is that we should maximize "orthogonality" subject to eliminating the possibility of obtaining invalid judgments.

We develop a methodology that attempts to alter the structure of $\mathbf{X}$ (the design matrix) to avoid these profiles and yet maintains as much orthogonality as possible. In order to do this, we first require a measure of orthogonality or, equivalently, lack of multicollinearity. The measure that appears most frequently in the statistics (experimental design/econometrics) literature [12] is the determinant of $\mathbf{X}^{\prime} \mathbf{X}\left(\left|\mathbf{X}^{\prime} \mathbf{X}\right|\right)$. If the independent variables (columns of $\left.\mathbf{X}\right)$ are standardized to zero mean and unit variance, then $\mathbf{X}^{\prime} \mathbf{X}$ is the correlation matrix of the (coded) attributes for the stimuli used in the estimation of the preference model. Here, $\left|\mathbf{X}^{\prime} \mathbf{X}\right|$ is in the interval $[0,1]$. If $\left|\mathbf{X}^{\prime} \mathbf{X}\right|=0$, one or more exact linear dependencies exist among the columns of $\mathbf{X}$. If $\left|\mathbf{X}^{\prime} \mathbf{X}\right|=1$, the columns of $\mathbf{X}$ are orthogonal.

We now wish to find an $\mathbf{X}^{*}$ such that $Z=\left|\mathbf{X}^{* \prime} \mathbf{X}^{*}\right|$ is maximized, subject to avoiding the user-specified unbelievable combinations of attribute levels. Later, other operationalizations of nonorthogonality will be used to assess various designs. Mitchell [15] [16] designed an exchange algorithm for the construction of "D-Optimal" designs to maximize $\left|\mathbf{X}^{\prime} \mathbf{X}\right|$. Our approach, developed independently, can be viewed as a modification of Mitchell's procedure in that (1) a different algorithm is utilized; (2) our procedure accommodates user specified constraints; and, (3) our procedure is tailor-made for conjoint designs. 
Table 1: Evaluation of specific designs for automobile example.

\begin{tabular}{|c|c|c|c|c|c|c|c|c|}
\hline \multirow[b]{3}{*}{ Mileage } & \multirow{2}{*}{\multicolumn{4}{|c|}{ Attributes and Levels }} & \multicolumn{4}{|c|}{ Specific Designs } \\
\hline & & & & & \multirow[b]{2}{*}{$\begin{array}{c}\text { Orthogonal } \\
\text { Design }\end{array}$} & \multirow{2}{*}{$\begin{array}{c}\text { Combinatorially } \\
\text { Optimized } \\
\text { Design }\end{array}$} & \multirow{2}{*}{$\begin{array}{c}\text { Orthogonal } \\
\text { Array Less } \\
\text { Infeasible } \\
\text { Profiles } \\
\end{array}$} & \multirow{2}{*}{$\begin{array}{c}\text { Optimal } \\
\text { Perturbation } \\
\text { of Orthogonal } \\
\text { Design }\end{array}$} \\
\hline & Price & $\begin{array}{l}\text { Maximum } \\
\text { Speed }\end{array}$ & $\begin{array}{c}\text { Car } \\
\text { Length }\end{array}$ & Roominess & & & & \\
\hline 20 & 10 & 85 & 10 & 2 & $r$ & & $r$ & $r$ \\
\hline 20 & 20 & 120 & 14 & 4 & $r$ & & 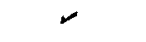 & $\sim$ \\
\hline 20 & 30 & 155 & 12 & 6 & $\sim$ & & $r$ & $\sim$ \\
\hline 30 & 10 & 120 & 12 & 4 & م & & $r$ & $\sim$ \\
\hline 30 & 20 & 155 & 10 & 6 & $\sim$ & & $r$ & $r$ \\
\hline 30 & 30 & 85 & 14 & 2 & - & & $r$ & $r$ \\
\hline 40 & 10 & 120 & 14 & 6 & $r$ & & & \\
\hline 40 & 20 & 155 & 12 & 2 & $r$ & & & \\
\hline 40 & 30 & 85 & 10 & 4 & r & & $r$ & $\sim$ \\
\hline 20 & 10 & 85 & 12 & 6 & $\boldsymbol{r}$ & & $\sim$ & $\sim$ \\
\hline 20 & 20 & 120 & 10 & 2 & $\sim$ & & $r$ & $r$ \\
\hline 20 & 30 & 155 & 14 & 4 & $\sim$ & & $r$ & $\sim$ \\
\hline 20 & 10 & 155 & 14 & 2 & $r$ & & & \\
\hline 30 & 20 & 85 & 12 & 4 & $r$ & & $\sim$ & $\sim$ \\
\hline 30 & 30 & 120 & 10 & 6 & $r$ & & $\sim$ & $r$ \\
\hline 40 & 10 & 155 & 10 & 4 & $r$ & & & \\
\hline 40 & 20 & 85 & 14 & 6 & - & & & \\
\hline 40 & 30 & 120 & 12 & 2 & $r$ & & $r$ & $\boldsymbol{v}$ \\
\hline 20 & 20 & 85 & 12 & 2 & & $r$ & & \\
\hline 20 & 10 & 85 & 10 & 4 & & $r$ & & \\
\hline 30 & 10 & 155 & 12 & 6 & & $r$ & & \\
\hline 30 & 30 & 85 & 14 & 4 & & $r$ & & \\
\hline 30 & 20 & 155 & 14 & 2 & & $r$ & & \\
\hline 30 & 20 & 155 & 10 & 2 & & $r$ & & \\
\hline 20 & 30 & 120 & 10 & 6 & & $r$ & & \\
\hline 40 & 20 & 120 & 12 & 6 & & $r$ & & \\
\hline 20 & 30 & 85 & 14 & 4 & & $r$ & & \\
\hline 20 & 10 & 85 & 12 & 4 & & $\sim$ & & \\
\hline 30 & 30 & 120 & 12 & 6 & & $\sigma$ & & \\
\hline 40 & 10 & 85 & 10 & 4 & & $\sigma$ & & \\
\hline 20 & 30 & 85 & 10 & 4 & & $r$ & & \\
\hline 30 & 30 & 155 & 10 & 2 & & $r$ & & \\
\hline 20 & 20 & 155 & 14 & 4 & & $r$ & & \\
\hline 40 & 30 & 85 & 14 & 4 & & $\sim$ & & \\
\hline 30 & 10 & 85 & 14 & 2 & & $r$ & & \\
\hline 40 & 30 & 85 & 10 & 2 & & $r$ & & \\
\hline 40 & 10 & 85 & 12 & 6 & & & & م \\
\hline 30 & 30 & 120 & 14 & 4 & & & & $r$ \\
\hline 30 & 10 & 120 & 14 & 6 & & & & $\sim$ \\
\hline 20 & 30 & 85 & 12 & 6 & & & & $r$ \\
\hline 30 & 10 & 155 & 12 & 2 & & & & $r$ \\
\hline \multicolumn{5}{|c|}{ Determinants } & 1 & .99 & .45 & .93 \\
\hline \multirow{2}{*}{\multicolumn{5}{|c|}{$\begin{array}{l}\text { Trace of Minor Product Inverse } \\
\text { Condition Number }\end{array}$}} & 5 & 5.01 & 7.16 & 5.15 \\
\hline & & & & & 1 & 1.07 & 2.21 & 1.29 \\
\hline
\end{tabular}

Note: $\sim=$ Profile is part of this design.

The value of $\left|\mathbf{X}^{\prime} \mathbf{X}\right|$ depends in part on the coding scheme used for $\mathbf{X}$. For example, $\left|\mathbf{X}^{\prime} \mathbf{X}\right|$ will be different for effects and dummy variable coded versions of the same orthogonal design in Table 1. Indeed, for nonorthogonal designs, $\left|\mathbf{X}^{\prime} \mathbf{X}\right|$ will differ for different versions of the same type (e.g., dummy variable) of coding. Furthermore, for orthogonal designs, $\left|\mathbf{X}^{\prime} \mathbf{X}\right|=1$ only for certain coding schemes. 


\section{The Procedure}

Thus, the optimization problem can be formally stated as:

$$
\operatorname{Max} Z=\left|\mathbf{X}^{\prime} \mathbf{X}\right|,
$$

subject to

$$
\mathbf{X}_{j} \text { representative for all } j \text {, }
$$

where $\mathbf{X}_{j}$ is the $j$ th row (profile) in the design matrix $\mathbf{X}$. That is, (1) is maximized over all experimental profiles that satisfy the user designated constraints which specify those profiles deemed to be nonrepresentative.

Expression (1) describes a constrained nonlinear integer programming problem that could be solved via a modified Balas [2] procedure or by Dakin's [5] modification of Land and Doig's [13] branch-and-bound procedure. Unfortunately, while such procedures would guarantee a globally optimal result, the cost of computing such solutions would be enormous. Complete enumeration would obviously be even more involved computationally.

We have devised a computationally more feasible approach using combinatorial optimization. We have programmed a modification of the Lin and Kernighan [14] algorithm. We attempt to generate a number of local optima by examining changes in the rows of $\mathbf{X}, m$ at a time. The steps of the modified algorithm are as follows:

1. Set $M=0$; select $m$ from $\left(1,2, \ldots, N^{*}\right\}$; set maximum number of solutions (MAXIT);

2. Generate all possible feasible row profiles and store them; generate an initial random feasible $\mathbf{X}$ from these feasible stored profiles;

3. Set $M=M+1$;

4. Evaluate objective function $(Z)$ and let $Z^{*}=Z$;

5. Generate a random map, that is, a random permutation of the first $N$ positive integers. This map indicates (randomly) the order in which rows will be changed $m$ at a time;

6. Try to improve-attempt to evaluate row changes in $\mathbf{X}, m$ at a time, according to the random map until either $\left(Z>Z^{*}\right)$ improves or all possible $m$ row changes are evaluated according to the map without improvement. If there is improvement, then set $Z^{*}=Z$, store the $\mathbf{X}$ solution that resulted in that $Z$, and go to step 5 . If no improvement results $\left(Z \leq Z^{*}\right)$, store $\mathbf{X}$ and the corresponding $Z$-value. If $M<M A X I T$, go to step 3; otherwise, output results and stop.

This procedure is much cheaper to run than the complete enumeration, branchand-bound, or zero-one programming approaches. Lin and Kernighan [14] estimated computer costs for the algorithm as a function of the number of parameters and described some of its interesting mathematical properties for the traveling salesman problem. A desirable feature of the algorithm is that it finds MAXIT local optimum solutions. This gives the analyst the opportunity to examine a number of "good" solutions, enabling him or her to examine the sensitivity of $Z$ to changes in various rows of $\mathbf{X}$ (e.g., changes in the stimulus profiles).

\section{The Example Revisited}

Consider, once again, the orthogonal design in Table 1 . At the bottom of the table is the $Z$ value, expression (1), along with two otiner measures often used to detect multicollinearity, $\operatorname{tr}\left[\left(\mathbf{X}^{\prime} \mathbf{X}\right)^{-1}\right]$ and the condition number [3] [11]. 
The combinatorial optimization methodology was applied to the automobile problem with a coding scheme which used the actual values. The result is shown in the second specific design column in Table 1 . The value of the objective function for this design is .99 , extremely close to the orthogonal design's value of 1 . The other efficiency measures also come very close to those of the orthogonal design.

How good is this solution? Can one do as well by utilizing some of the simple ad hoc remedies (options b, c, d, e)? Given the multiple attribute nature of the constraints, we do not evaluate option $g$ since the number of row profiles will increase dramatically. Also, it is impossible to create an alternative orthogonal array by recoding or generating another design with these constraints. However, suppose one were simply to delete rows $7,8,13,16$, and 17 in the orthogonal design. The third design column in Table 1 presents the corresponding statistical measures. As can be easily seen, the various orthogonality measures all differ greatly from those of the orthogonal design. Thus, this is not a very appealing solution.

One could also try to change profiles $7,8,13,16$, and 17 to be believable. There are eight attribute level changes for each of profiles 7,13 , and 17 and four for 8 and 16. Thus, there are $8192\left(8^{3} \times 4^{2}\right)$ possible modifications. Rather than search them all, we executed a random search routine to find the best modification. The fourth design column in Table 1 presents the best one found. We were able to generate a representative solution doing this, but could not match the efficiency of the one in the second column. More importantly, the efficiency measures changed quite significantly depending on how the original design was altered.

\section{DISCUSSION}

Recognizing the potential difficulties inherent in nonrepresentative stimuli, this paper proposed a combinatorial optimization procedure for developing nearly orthogonal designs with representative stimuli. A simple automobile preference example was used to illustrate the method. In this case, we showed that a variety of ad hoc methods did not produce designs that are as orthogonal as the optimal one we derived.

Before the proposed procedure is applied, however, some of its limitations warrant special mention. First, the proposed methodology produces designs that may be only locally optimal. However, allowing local optimality avoids the computational burden of branch-and-bound and complete enumeration methods. In implementing the proposed procedure, we do urge that a user execute the program several times (MAXIT), each with a different randomly chosen starting $\mathbf{X}$, to confirm the optimality of the final solution. In fact, MAXIT is user-specified. While our procedure does not always guarantee a globally optimal result, setting MAXIT $\geq 10$ in our problem always resulted in obtaining the same maximum value (although corresponding designs did differ) for $m=1$ or 2 . Obviously, the larger one makes $m$ and MAXIT, the higher the probability of finding the globally optimal result. On the other hand, it also increases computational time and cost. Our experiences on manipulated sample $m$ in sample problems suggest that $m=2$ works best. Thus, it is our default option. See [14] for a similar view.

Our automobile illustration was based on a coding scheme in which the actual attribute values were used. Although this is a common approach, it is far from universal. One of the attractive features of our algorithm is that it can accommodate any coding scheme the user wishes with simple modifications. (The user must create a subroutine which translates attribute levels to the desired coding scheme.) 
Future research should pursue both applications and theoretical developments. Of utmost importance would be the clarification of the variety of specific cognitive effects that nonrepresentative stimuli may have on responses. Experimental manipulations of stimuli could be performed in conjunction with protocol analyses and test theory based analyses to examine these issues. Such understanding would isolate appropriate situations for our algorithm.

Furthermore, whatever impact a nonrepresentative or unbelievable stimulus has would likely be a function of the degree of nonrepresentativeness inherent in the stimulus. Nonrepresentativeness is more likely a continuum than a dichotomy. The functional nature of the relationship between the degree of nonrepresentativeness and the deviation of the response from the true preference model is an open question. [Received: April 7, 1989. Accepted: March 13, 1990.]

\section{REFERENCES}

[1] Addelman, S. Orthogonal main-effect plans for asymmetrical factorial experiments. Technometrics, 1962, 4, 21-46.

[2] Balas, E. An additive algorithm for solving linear programs with zero-one variables. Operations Research, 1965, 13, 517-546.

[3] Belsley, D. A., Kuh, E., \& Welsch, R. E. Regnession diagnostics. New York: Wiley, 1980.

[4] Cattin, P., \& Wittink, D. R. Commercial use of conjoint analysis: A survey. Journal of Marketing, 1982, 46, 44-53.

[5] Dakin, R. J. A tree search algorithm for mixed integer programming problems. Computer Journal, 1965, 8, 250-255.

[6] Green, P. E. On the design of choice experiments involving multifactor alternatives. Journal of Consumer Research, 1974, 1, 61-68.

[7] Green, P. E., \& Rao, V. R. Conjoint measurement for quantifying judgmental data. Journal of Marketing Research, 1971, 8, 355-363.

[8] Green, P. E., \& Srinivasan, V. Conjoint analysis in consumer research: Issues and outlook. Journal of Consumer Research, 1978, 5, 103-123.

[9] Green, P. E., \& Tull, D. R. Research for marketing decisions (4th ed.). Englewood Cliffs, NJ: Prentice-Hall, 1978.

[10] Hoffman, P. J. Cue-consistency and configurality in human judgment. In B. Kleinmuntz (Ed.), Formal representation of human judgment. New York: Wiley, 1968.

[11] Johnston, J. Econometric methods (3rd ed.). New York: McGraw-Hill, 1983.

[12] Judge, G. G., Griffiths, W. E., Hill, R. C., \& Lee, T. The theory and practice of econometrics (2nd ed.). New York: Wiley, 1985.

[13] Land, A. H., \& Doig, A. An automatic method of solving discrete programming problems. Econometrica, 1960, 28, 497-520.

[14] Lin, S., \& Kernighan, B. An effective heuristic algorithm for the traveling salesman problem. Operations Research, 1973, 21, 498-516.

[15] Mitchell, T. J. An algorithm for the construction of 'D-Optimal' experimental designs. Technometrics, 1974, 16, 203-210.

[16] Mitchell, T. J. Computer construction of 'D-Optimal' first order designs. Technometrics, 1974, 16, 211-220.

[17] Riedesel, P. L. Conjoint analysis is a worthwhile tool, but be sure data are valid. Marketing News, September 13, 1985, 36-43.

Joel H. Steckel is an Associate Professor of Marketing at the Stern School of Business, New York University. He holds a B.A. in mathematics from Columbia University and an M.B.A., M.A., and Ph.D. from the Wharton School of the University of Pennsylvania. His current research interests include the modeling of managerial and consumer decision processes, particularly as they involve groups. Dr. Steckel's most recent publications have appeared in Journal of Consumer Research, Psychometrika, Marketing Science, Psychological Bulletin, and Journal of Business and Economic Statistics among others.

Wayne S. DeSarbo is the S.S. Kresge Professor of Marketing and Statistics, Graduate School of Business Administration, University of Michigan. He holds a B.S., M.A., and Ph.D. from the University of Pennsylvania as well as a master's degree from Yale University. His research interests center 
on psychometrics and its application to marketing. Dr. DeSarbo has published over 75 articles in a wide variety of professional journals.

Vijay Mahajan is the James L. Bayless/ENSTAR Chair Professor of Business Administration, Graduate School of Business Administration, University of Texas at Austin. Dr. Mahajan received a B.Tech. in chemical engineering from the Indian Institute of Technology, Kanpur, India, and an M.S. in chemical engineering and Ph.D. in management from the University of Texas at Austin. He has written and published extensively on product diffusion, marketing strategy and research. He is the departmental editor of Planning and Forecasting for Management Science. 